\title{
IDENTIFICATION OF BRAIN LESIONS IN NEUROPSYCHIATRIC SYSTEMIC LUPUS ERYTHEMATOSUS BY MAGNETIC RESONANCE SCANNING
}

\section{W. JOSEPH McCUNE, ANNE MACGUIRE, ALEX AISEN, and STEPHEN GEBARSKI}

Cranial magnetic resonance imaging in 28 systemic lupus erythematosus patients who had experienced 30 acute neuropsychiatric events showed focal brain lesions in 16 of 30 events $(53 \%)$ and low brain volume (atrophy) in 20 of 30 events $(67 \%)$. Definite focal lesions were significantly more frequent in patients with clinically localized neurologic deficits $(8$ of $8,100 \%)$, or seizures $(5$ of $6,83 \%$ ) than in patients without such localizing signs ( 3 of $16,19 \%)$. Many of these lesions were occult on intravenous contrast-enhanced $x$-ray computed tomography. In 2 patients, lesions in gray matter resolved within 2 or 3 weeks, in association with clinical improvement. Magnetic resonance imaging is an important technique for detecting the extent of brain injury in cerebral lupus.

Significant neuropsychiatric events occur in as many as $70 \%$ of patients with systemic lupus erythematosus (SLE) $(1,2)$. Despite the frequent occurrence of neuropsychiatric lupus (NPSLE), our understanding of these events has recently been said to be comparable with our understanding of lupus nephritis 30 years ago (1). A useful classification scheme is needed $(3,4)$. In 2 large studies, pathologic findings at autopsy correlated poorly with clinical events, and showed vasculitic le-

From the Departments of Medicine and Radiology, University of Michigan Hospitals, Ann Arbor.

Supported in part by gifts from the Michigan Lupus Foundation and the Northwest Ohio Lupus Foundation.

W. Joseph McCune, MD; Anne MacGuire, MD; Alex Aisen, MD; Stephen Gebarski, MD.

Address reprint requests to W. Joseph McCune, MD, University of Michigan Hospitals, 3918 Taubman Center, Ann Arbor, MI 48109-0358.

Submitted for publication August 4, 1986; accepted in revised form June $29,1987$. sions typical of extracranial SLE in only a fraction of patients $(5,6)$. These studies were complicated by the failure to distinguish between "primary" neuropsychiatric events that presumably result from the direct effect of active SLE on the nervous system and "secondary" events that result indirectly from SLE-induced dysfunction of organs outside the nervous system (e.g., uremia, hypertension), or from the side effects of drug therapy for SLE (4).

Before the introduction of magnetic resonance imaging (MRI), there was no satisfactory method of consistently identifying localized cerebral lesions in NPSLE. The few focal lesions detected by $x$-ray computed tomography (CT) have correlated well with clinical findings, but such abnormalities were often not demonstrated in patients with clinical evidence of recent strokes or seizures (7-9). Other imaging methods used to identify brain lesions in NPSLE have limitations in sensitivity (angiography), specificity (oxygen 15 positron emission tomography) (10), or in both (radionuclide brain scanning) (11-13).

MRI offers several theoretical advantages over CT when applied to brain imaging; notably, better intrinsic resolution of various normal and pathologic tissues without the need for intravenous contrast material, lack of beam hardening and scatter artifacts from surrounding bone, and availability of images in multiple orthogonal planes without manipulation of the patient (14). Using current technology, the improved sensitivity of MRI compared with that of CT in the central nervous system (CNS) has been demonstrated in the majority of patients with noncalcified nonhemorrhagic brain lesions $>3 \mathrm{~mm}$ in diameter (15-17). Previous studies that were limited to the description of only those patients who had abnormal results on MRI 
have suggested improved sensitivity compared with CT in NPSLE $(18,19)$.

We obtained cranial MRI scans in 28 patients with 30 recent neuropsychiatric events that were associated with SLE; 1 patient was studied on 3 different occasions. Our findings suggest that MRI detects more local abnormalities in specific clinical subsets of patients with NPSLE than does CT. Because MRI was more sensitive than CT only in the presence of specific clinical findings, we conclude that the additional abnormalities detected were real and not artifacts.

\section{PATIENTS AND METHODS}

Patients. Patients were included in this study only if they fulfilled all of the following criteria: 1) Admission to the University of Michigan Hospital and/or had received outpatient care in the rheumatology clinic. 2) Documentation of 4 American Rheumatism Association (ARA) criteria for SLE (20). The ARA criterion of neuropsychiatric disease was considered to be fulfilled only if a seizure or psychosis was documented in the absence of a secondary cause as defined below. 3) A significant change in neuropsychiatric status potentially attributable to SLE, its complications, or treatment, within the preceding 4 weeks (acute disease). 4) Performance of a formal neurologic examination.

After permission was obtained from the patient's attending physician, all patients with acute NPSLE known to the investigators who satisfied the above criteria over a 16-month period were offered the opportunity to have MRI scanning performed. Patients for whom MRI scanning was contraindicated (pregnancy, metal clips near great vessels, or an acute medical problem that rendered them too unstable to undergo a scan) were excluded. Written consent was obtained prior to MRI. Further studies, including CT, electroencephalogram, and cerebrospinal fluid (CSF) analysis, were carried out at the discretion of the attending physicians and were not specifically performed as part of the protocol.

Records of all patients from the time of diagnosis of SLE were reviewed in their entirety. Clinical, laboratory, and imaging data were tabulated in chart form by an investigator (AM) who was unaware of the results of MRI scan interpretations. Particular attention was paid to previous neurologic events, evidence of vasculitis, vasoocclusive disease, or coagulopathies. The following data were specifically sought: 1) the age at diagnosis (4 ARA criteria for SLE or, in the absence of detailed records, the age at onset of obvious multisystem disease that was typical of SLE); 2) the presence of vasculitis, either proven by biopsy, or obvious cutaneous infarction or purpura; 3) thrombotic episodes, including lower extremity thrombophlebitis, pulmonary embolism, angiographic and/or biopsy-proven thrombosis in other organs; 4) prolongation of the partial thromboplastin time (PTT; upper limit of normal 28 seconds) at any time during the clinical course in the absence of anticoagulation therapy, disseminated intravascular coagulation, or an inherited coagulopathy. In addition, the prednisone "index" was said to be the integral, over time, of all doses of prednisone documented in the chart, assuming that the patient took the recorded dose constantly until a change was recorded. Laboratory values, including CSF analysis, complement CH50 determinations using the modified method described by Kent and Fife (21), and serum antibodies to doublestranded DNA by the Farr or Crithidia assays, were recorded if determined within 4 weeks of the event on the date closest to the event studied. All previous neurologic events were recorded in detail.

Neuropsychiatric events were classified according to the following criteria (based on clinical findings in the 4 weeks preceding MRI). Group I: Group 1 included all patients with a new localizing physical finding on neurologic examination that was attributable to a brain lesion. Group 2: Group 2 was composed of all patients with either witnessed or self-reported seizures that were corroborated by EEG findings, who did not qualify for inclusion in group 1. Group 3: Group 3 included all other patients with significant neuropsychiatric events who did not meet criteria for inclusion in group 1 or group 2 . Included were patients with progressive organic brain syndromes, psychoses, headaches, or meningeal signs that prompted hospital admission, selfreported localizing neurologic deficits (visual or motor) not accompanied by corresponding findings on neurologic examination, drug toxicity, and transverse myelitis.

Neurologic events were further classified as primary (presumably resulting from active SLE affecting the brain) or secondary (possibly resulting indirectly from SLE activity that is primarily affecting organs outside of the CNS) if 1 or more of the following critiera were present: uremia (blood urea nitrogen $>100$ or dialysis), hypertension (diastolic blood pressure $>110 \mathrm{~mm} \mathrm{Hg}$ ), hypoxia (decrease in $\mathrm{Po}_{2}$ to $<60$ ), culture-proven systemic or CNS infection, serious electrolyte imbalances, probable side effects of drugs, or thrombocytopenia (platelet count $<50,000 / \mathrm{mm}^{3}$ ) accompanied by CNS hemorrhage (4).

MRI. MRI was performed utilizing a Diasonics MT/S system employing a super-conducting 0.5 tesla (T) magnet operating at $0.35 \mathrm{~T}$. All images were obtained with multislice. spin-echo technique imaging hydrogen nuclei; $7 \mathrm{~mm}$-thick sections at $10-\mathrm{mm}$ intervals were obtained, with $1.7 \times$ $1.7-\mathrm{mm}$ resolution. Axial imaging sequences with a pulse repetition time (TR) of $2,000 \mathrm{~ms}$ and echo time (TE) of 28 and $56 \mathrm{~ms}$ were performed on all patients. This sequence emphasizes spin-spin transverse relaxation time $\left(\mathrm{T}_{2}\right)$ abnormalities. Such emphasis is commonly referred to as $\mathbf{T}_{2}$ weighting. This sequence delivers 20 pairs of axial images in approximately 17 minutes. Initially, patients also received a second axial sequence with balanced (TR $1,000 \mathrm{~ms}$, TE 28 and 56 $\mathrm{ms})$ technique, that is, without dominant weighting for spin lattice relaxation time $\left(T_{1}\right)$ or for $T_{2}$. This sequence delivers 10 axial images in approximately 5 minutes. This balanced technique did not prove useful and was replaced by a $\mathrm{T}_{1}$-weighted (TR $500 \mathrm{~ms}$, TE $28 \mathrm{~ms}$ ) axial sequence which delivers 10 axial image pairs in approximately 8 minutes. Questionable abnormalities seen using the $\mathrm{T}_{2}$-weighted sequence would result in the patient receiving a corroborative coronal sequence with relative $T_{2}$ weighting (TR $1,500 \mathrm{~ms}$, TE 28 and $56 \mathrm{~ms}$ ) delivering 15 coronal sections in approximately 13 minutes. 
Table 1. Acute neuropsychiatric events in 28 patients with systemic lupus erythematosus

\begin{tabular}{lc}
\multicolumn{1}{c}{ Classification } & No. of events \\
\hline Group 1 (focal events) & 8 \\
Group 2 (seizures) & 4 \\
Simple & 2 \\
Complex & 6 \\
Group 3 (other events) & 1 \\
Organic brain syndromes & 4 \\
Psychosis & \\
Headaches or meningeal signs & 3 \\
Visual or motor symptoms (normal results on & 1 \\
$\quad$ neurologic examination) & 1 \\
Plaquenil toxicity & 30 \\
Transverse myelitis & \\
\hline Total &
\end{tabular}

CT. Computed tomography scans obtained early in the study were by an EMI 1005 dedicated head scanner updated to 1010 performance. CT scans obtained later were by General Electric 8800 or 9800 units. Intravenous contrast enhancement was used in most patients, and was omitted in patients only because of a history of contrast sensitivity, renal insufficiency, and/or emergency indications for CT, generally, clinical suspicion of intracranial hemorrhage, requiring non-contrast-enhanced CT. Nineteen patients underwent $\mathrm{CT}$.

MRI/CT interpretation. All MRI scans were interpreted at the time of acquisition by 2 investigators (AA, WJM), and additional sequences were obtained if needed, using the method described above. At the conclusion of this study, all MRI sequences, both $T_{2^{-}}$and $T_{1}$-weighted, were interpreted in a blinded manner (5 normal controls intermixed, the interpreter unaware of the patient's medical diagnosis or previous scan interpretations) by a neuroradiologist (SG). Definite circumscribed abnormalities were considered to be present if they were positively identified in any pulse sequence. The only information provided at the time of the neuroradiologist's interpretation was the age and sex of the patient. CT was interpreted by the neuroradiologist without identification of the corresponding MRI scan, again in the blinded manner described above.

MRI and CT interpretations were recorded separately, on a standardized form during blinded reading. Circumscribed parenchymal lesions were classified as definite or equivocal, and their size, location (peripheral, junctional, or periventricular), and the dominant type of brain tissue

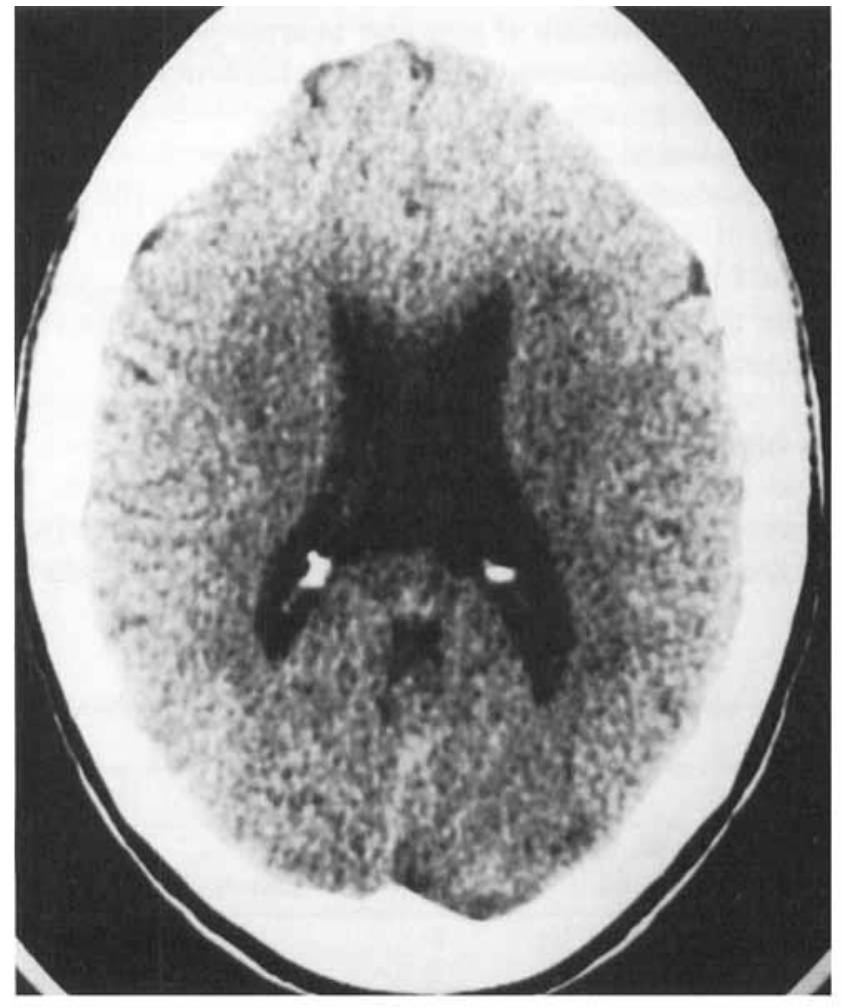

A

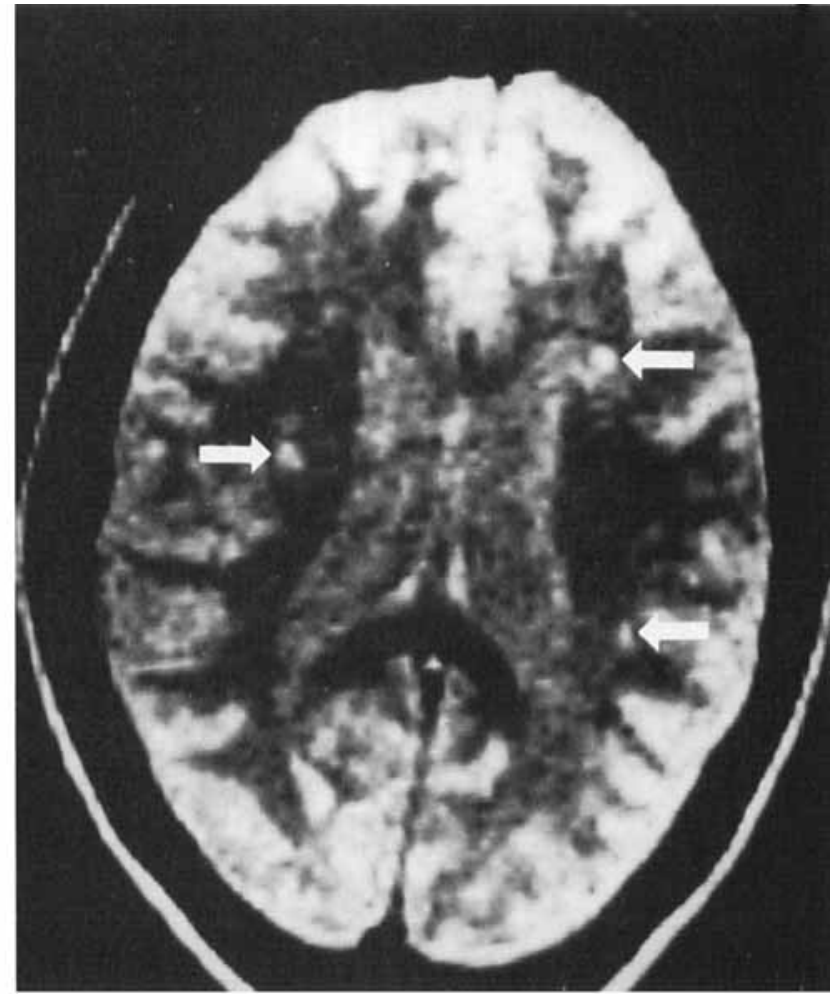

B

Figure 1. A, Computed tomogram of a 52-year-old woman with lupus and partial complex seizure activity, showing no abnormalities. B, Cranial magnetic resonance scan obtained at the same time and at the same level, showing new appearance of multiple white matter lesions (arrows) which were absent on a magnetic resonance scan obtained 1 year previously. 


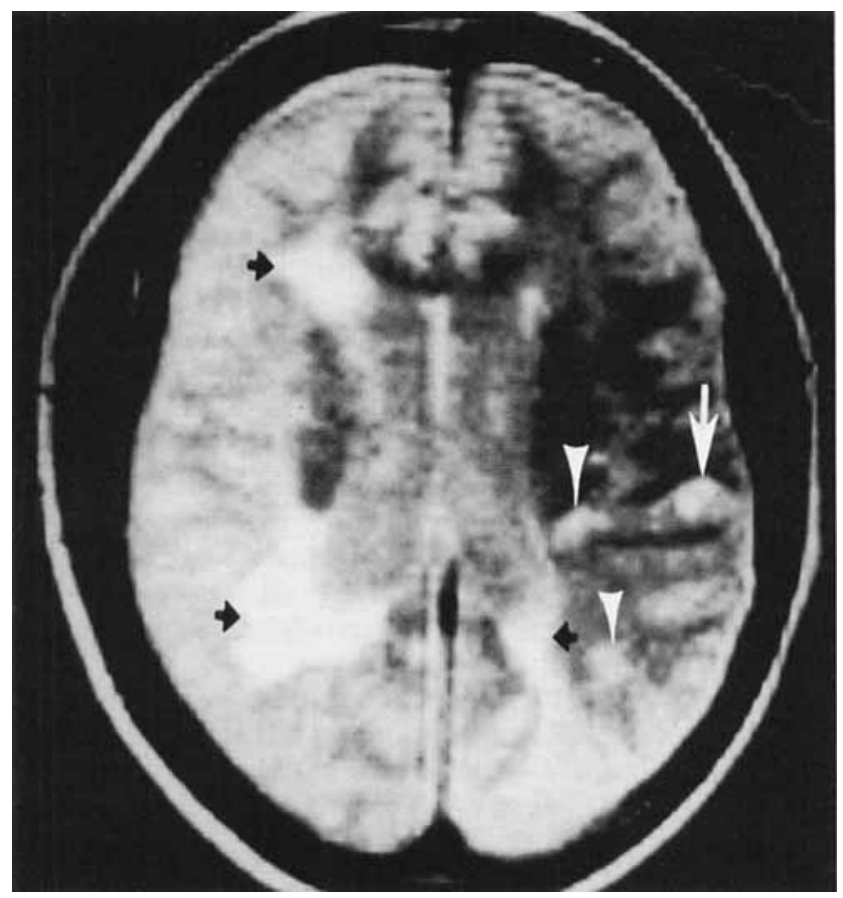

Figure 2. Magnetic resonance scan of a 36-year-old woman with multiple strokes and a seizure disorder. There are multiple periependymal white matter lesions (black arrows), a new junctional lesion that predominantly involves gray matter (white arrow), and deep white matter lesions (arrowheads).

affected (white matter, gray matter, or both) were identified. The degree of low brain volume for age, or atrophy, was assessed in 4 areas: supratentorial and infratentorial superficial brain tissue, and supratentorial and infratentorial deep brain tissue. In each location, the degree of atrophy was subjectively ranked from 0 (normal) to 3 (very severe). For each patient, the atrophy score was determined as the sum of the numerical values assigned to each of the 4 regions. Thus, a normal atrophy score was 0 , and the maximum possible abnormality score was 12 .
Statistical methods. Results were analyzed by Fisher's exact test, with Yates' correction where deemed appropriate. Grouped numerical results were compared by logistic regression. For comparison of numerical results in the patient with multiple events, the second event was arbitrarily selected.

\section{RESULTS}

All of the normal subjects ( 5 individuals) had normal scan results. Thirty acute events were studied in 28 patients ( 8 group 1 events, 6 group 2 events, 16 group 3 events) (Table 1). Twenty-three events were primary and 7 were secondary. Only 3 of the events were accompanied by additional neurologic findings which, if present in isolation, would have resulted in assignment to a different category of neurologic event (e.g., group 2, rather than 1 or 3 ); 27 events were in 1 specific category, and did not have elements of an additional category as a confounding feature. Focal events occurred more than 1 month prior to entry into the study in one group 2 patient and one group 3 patient. Characteristic images obtained from study patients are shown in Figures 1 and 2.

Relationship of previous neuropsychiatric events to group assignment. There was a tendency for previous neuropsychiatric events to be classified into the same clinical group as the current neuropsychiatric events studied in individual patients. Thus, in the events studied, 17 patients had previous recorded events which would have been assigned to the same group, whereas prior events which would have been classified differently occurred in only 10 patients.

MRI identification of circumscribed brain lesions in blinded readings. Among the 30 studies obtained, it was possible to definitely judge focal lesions to be present or absent in 28 (Table 2). Two studies (one from a group 2 patient and one from a group 3 patient)

Table 2. Identification of circumscribed brain lesions by magnetic resonance imaging in neuropsychiatric systemic lupus erythematosus patients

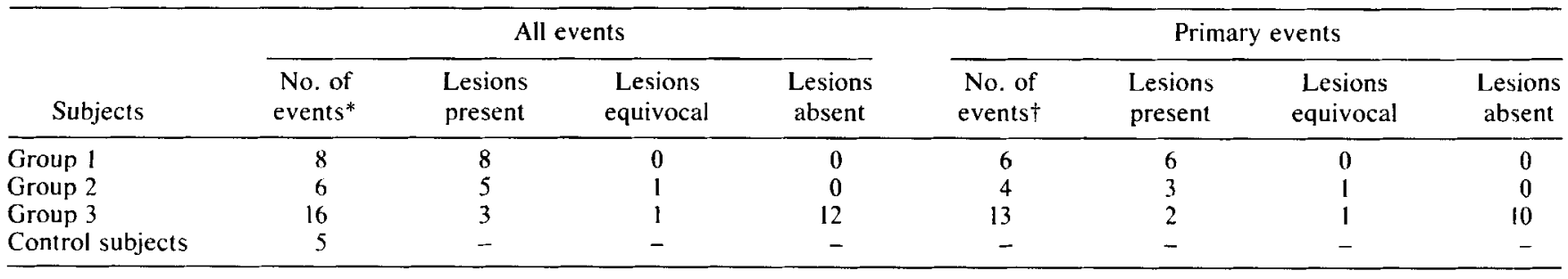

* Comparing number of patients with positive results versus number with negative results, $P<0.01$, group 1 versus group 3 and group 2 versus group 3.

$†$ Comparing number of patients with positive results versus number with negative results. $P<0.05$, group $\mid$ versus group 3 . 
showed equivocal abnormalities, possibly because of technical limitations of MRI, and are excluded from this part of the analysis. Eight of 8 group 1 scans (100\%) and 5 of 5 group 2 scans $(100 \%)$ had positive results, compared with 3 of 15 group 3 scans (20\%) (group 1 versus group $3 P<0.01$; group 2 versus group $3 P<$ $0.01)$ When only a single event was analyzed for each patient (the chronologically latest event was arbitrarily selected), these correlations again had a slightly greater level of significance. When only patients with neuropsychiatric events classified as primary (that is, without known possible etiologies for CNS disease other than SLE) were analyzed, there was again a significant difference between group 1 and group 3 patients.

The majority of the lesions were quite obvious to all observers. All lesions previously noted by unblinded observers were recognized by the blinded observer, who noted 1 additional lesion in group 1, 1 in group 2, and 2 in group 3 . There were 6 lesions confined to gray matter, 6 lesions confined to white matter, and 4 lesions that overlapped. In group 1, 6 of 8 scans showed lesions in the appropriate hemisphere and in an anatomic location that corresponded to clinical findings. We were unable to differentiate abnormalities in patients in group 2 (seizures) from those in group 1 (focal signs) on the basis of the location of lesions.

Identification of brain atrophy by MRI scanning. Minor degrees of atrophy affecting the superficial brain structures above and below the tentorium were common, but severe atrophy or atrophy of deep structures was uncommon.

Atrophy was identified on 20 scans (67\%), with a mean score of 1.5 (range 0-12) in affected patients. Superficial atrophy was noted on 20 scans; atrophy of deep structures was noted on 2 scans $(P<0.01)$. Atrophy was noted in 6 of 8 group 1 scans (75\%), 5 of 6 group 2 scans (83\%), and 9 of 16 group 3 scans (56\%). Atrophy and/or a focal abnormality was present in 23 scans. The atrophy score was low in all patients, and reflected, in part, the localization of most findings to the supratentorial superficial structures (range of positive scores 1-3) and absence of findings in the 3 other areas evaluated. In all instances, patients with atrophy had diffuse involvement of the region evaluated, including areas not containing focal lesions.

Relationship of clinical and laboratory parameters to MRI scan interpretations. The most significant parameter associated with MRI scan findings by multivariate analysis was age. Patients with focal lesions were significantly older than unaffected patients, and patients with atrophy were younger than those without atrophy, as shown in Table 3. Review of the findings depicted in Table 3 suggests a number of other possible associations which did not achieve statistical significance, including association between vascular thrombosis and focal lesions, and association of vascular thrombosis, prolongation of the PTT, and a high degree of prednisone intake with cerebral atrophy. There was no relationship of imaging findings to levels of anti-DNA antibodies, CH50, or CSF protein, glucose, or cell count.

CT. CT scans were obtained for 19 patients. Focal lesions were identified in 5 of 19 CT scans $(26 \%)$, compared with 12 of 19 MRI scans $(63 \%)$. CT and MRI were obtained in close temporal relationship, with an average time interval of 40 hours. One patient had equivocal abnormalities on both MRI and CT. There was substantial consistency between MRI and CT results in the identification of focal lesions in group 3 patients, since results of almost all scans were negative. In contrast, focal lesions were identified by CT in only 2 of 6 patients in group 1 and 2 of 4 patients in group 2, compared with 10 of 10 positive corresponding MRI scans in groups 1 and 2. Thus, in patients at greatest risk for focal lesions (group 1 and group 2), 4 of $10 \mathrm{CT}$ scans, compared with 10 of $10 \mathrm{MRI}$ scans, gave positive results $(P<0.05)$. All lesions identified on CT were also identified on MRI. As shown in Table 4, lesions which were identified by MRI were also seen by $\mathrm{CT}$ in 1 of 5 scans with isolated white matter

Table 3. Correlation of clinical and laboratory parameters with magnetic resonance imaging findings in 28 patients with neuropsychiatric systemic lupus erythematosus*

\begin{tabular}{|c|c|c|c|c|}
\hline & \multicolumn{2}{|c|}{ Focal lesions } & \multicolumn{2}{|c|}{ Atrophy } \\
\hline & Present & Absent & Present & Absent \\
\hline Median age & $36 \dagger$ & 28 & $28.5 \dagger$ & 51 \\
\hline $\begin{array}{l}\text { History of vascular } \\
\text { thrombosis }\end{array}$ & $5 / 14$ & $1 / 13$ & $6 / 19$ & $0 / 9$ \\
\hline History of vasculitis & $4 / 14$ & $2 / 13$ & $6 / 19$ & $0 / 9$ \\
\hline $\begin{array}{l}\text { Prednisone index }>25 \mathrm{gm} \\
\quad \text { (lifetime intake) }\end{array}$ & $4 / 1$ & $6 / 13$ & $7 / 19$ & $3 / 9$ \\
\hline $\begin{array}{l}\text { History of prolonged PTT } \\
\text { (normal 13-28) } \\
\text { Cerebrosninal fluid }\end{array}$ & $5 / 13$ & $2 / 11$ & $7 / 18$ & $1 / 7$ \\
\hline White cells $>5 / \mathrm{mm}^{3}$ & $1 / 8$ & $1 / 6$ & $8 / 17$ & $1 / 6$ \\
\hline Protein $>40 \mathrm{mg} \%$ & $2 / 9$ & $1 / 5$ & $2 / 9$ & $2 / 6$ \\
\hline $\begin{array}{l}\text { Serum CH50 }<100 \text { (normal } \\
104-188 \text { ) }\end{array}$ & $5 / 10$ & $4 / 8$ & $7 / 13$ & $4 / 5$ \\
\hline $\begin{array}{l}\text { Serum DNA binding }>25 \% \\
\quad(\text { normal } \pm 3 \text { SD } 0-25)\end{array}$ & $5 / 11$ & $6 / 9$ & $6 / 14$ & $5 / 6$ \\
\hline
\end{tabular}

* Except for the age parameter, numbers are the no. found/no. of patients tested. PTT $=$ partial thromboplastin time. $\dagger P<0.05$ versus absent. 
involvement (20\%), and 1 of 4 scans with isolated gray matter involvement (25\%), compared with 3 of 4 scans with involvement of both white matter and gray matter (75\%). Lesions that involved both white and gray matter tended to be larger than those that did not.

In paired CT and MRI scans, blinded subjective evaluation of atrophy (performed on separate occasions without patient identification) showed an atrophy score $\geq 1$ in 6 of 17 CT scans compared with 11 of 17 MRI scans ( $P$ not significant). As in MRI evaluations, the degree of atrophy seen by CT was mild.

Transient focal lesions. In 2 patients, 1 with aphasia and hemiparesis and 1 with a seizure which occurred while the patient was hypertensive and uremic, MRI demonstrated unequivocal single focal lesions which, on repeat MRI scans obtained within 2-3 weeks, appeared to have resolved. Both lesions were located primarily in gray matter and were not demonstrated, even in retrospect, by emergency non-contrast-enhanced CT. In both patients, clinical symptoms resolved in parallel with resolution of abnormalities on the scans.

\section{DISCUSSION}

In 28 patients with active neuropsychiatric SLE, MRI detected focal lesions in 16 of 30 scans and atrophy in 20 of 30 scans, with some abnormality seen on 23 of 30 scans. In this series, the presence or absence of circumscribed brain lesions correlated well with clinical evidence of either focal neurologic deficits or seizures, and the correlation was better than that obtained using CT. Although the classification scheme of clinical events was designed so that patients with multiple manifestations were more frequently assigned to groups 1 and 2, most patients did not have

Table 4. Characteristics of focal lesions identified in patients who had undergone both magnetic resonance imaging (MRI) and computed tomography (CT) scans

\begin{tabular}{|c|c|c|c|}
\hline Location of lesion & $\begin{array}{l}\text { No. of } \\
\text { patients }\end{array}$ & $\begin{array}{l}\text { No. }(\%) \text { of } \\
\text { patients with } \\
\text { lesions } \\
\text { detected by } \\
\text { MRI }\end{array}$ & $\begin{array}{l}\text { No. (\%) of } \\
\text { patients with } \\
\text { lesions } \\
\text { detected by } \\
\text { CT }\end{array}$ \\
\hline $\begin{array}{l}\text { Isolated to white or } \\
\text { gray matter }\end{array}$ & 9 & $9(100)$ & $2(22)$ \\
\hline White matter only & 5 & $5(100)$ & $1(20)$ \\
\hline Gray matter only & 4 & $4(100)$ & $1(25)$ \\
\hline Overlapping lesions & 4 & $4(100)$ & $3(75)$ \\
\hline Total & 13 & $13(100)$ & $5(38)$ \\
\hline
\end{tabular}

overlapping acute symptoms, as shown in Table 3 , and it appears unlikely that bias toward including patients with more severe disease in these groups was solely reponsible for the marked increase in the occurrence of focal lesions in groups 1 and 2 compared with group 3 . Our data suggest that the presence of focal abnormalities seen on MRI is characteristic of the occurrence of seizures and strokes in SLE patients, even when the accompanying CT result is negative. An underlying pathophysiologic process which can produce localized brain abnormalities, with or without additional diffuse brain injury, should be sought.

Sequential observation of 2 reversible lesions, each of which was not detectable by CT, showed direct correlation between resolution of abnormalities seen on MRI and clinical improvement. This correlation suggests that either the abnormalities detected by MRI may improve in many patients because of partial or complete reversal of parenchymal injury, accompanied by clinical improvement, or that the lesion does not heal, but becomes undetectable by current MRI techniques concomitantly with recruitment of alternate neuronal pathways to restore clinical function. The excellent correlation of focal lesions seen on MRI with acute events (limited to only the 4 weeks preceding MRI) without consideration of prior events may be related, in part, to resolution of focal lesions which might have been transiently present in those patients with group 3 events who had previous events classified as group 1 or group 2.

In our series, lesions confined either to gray matter or to white matter tended to be smaller than lesions involving both gray and white matter. This may explain why overlapping lesions were most reliably detected by CT. Alternatively, isolated gray or white matter lesions might have different imaging characteristics due to a different type of tissue injury.

Cerebral atrophy was a frequent MRI finding, and was usually confirmed by CT. Atrophy tended to be superficial rather than deep, and ventricular enlargement was distinctly uncommon. The majority of patients had findings confined to the superficial, supratentorial region. The mean score of 1.5 (on a scale of $0-3$ ) is representative of mild-to-moderate abnormality in this region, with only a minimal contribution from the 3 other areas scored. It was the subjective judgment of our blinded observer (SG), as well as the 2 other investigators (AA, WJM) that the findings, in general, were mild, although definite and easily distinguished from those on the scans of the normal controls. Although there was a tendency for atrophy to 
occur more frequently in patients in group 1 and group 2 , as well as in patients who also had focal lesions seen on MRI, these associations reached only marginal statistical significance. Atrophy has previously been thought, by some investigators (22-25), to be related to steroid therapy, and there was a tendency for it to be associated with higher steroid doses in this study.

When both techniques were used, more lesions were identified by MRI than by CT. The comparison may not reflect findings detected using optimal CT techniques, because practical considerations precluded obtaining studies with and without contrast in all patients. However, because CT was ordered by the attending physician only when it was thought to be clinically indicated (and was not routinely performed as part of the protocol), it is possible that patients whose physicians considered them to be more severely ill were more likely to undergo CT. Therefore, they might have been more likely to have positive findings than those patients for whom CT was not ordered. When emergency studies were performed, CT was always obtained first and MRI was obtained only when the patient was stable, usually $12-48$ hours later. It is possible that if CT scans had been obtained longer after the acute event, additional findings might have been noted. It is also possible that findings on some MRI scans might have been negative if they had been obtained earlier.

Although assessments of the specificity of our findings for lupus-mediated CNS damage will require additional control subjects without neuropsychiatric lupus as well as steroid-treated control subjects without lupus, the very low incidence of focal lesions in group 3 patients, who were frequently treated with steroids, suggests that focal lesions would be at least equally uncommon in a control population. There are no published data regarding MRI of steroid-treated patients who do not have central nervous system disease. Interpretation of findings of cerebral atrophy, which correlated positively with estimated prednisone dosage, will be aided by such studies.

Our findings suggest that the presence or absence of focal lesions in lupus patients with acute neuropsychiatric events is currently best determined by MRI. Presently, abnormal findings would appear to be most useful in patient management when clinical suspicion of a lesion in a specific location is confirmed, since the significance of asymptomatic lesions and the incidence of focal abnormalities in lupus patients who do not have clinical evidence of neurologic dysfunction is currently unknown.
Although MRI is quite sensitive, its specificity is limited. In most patients, it would not be possible to suggest a diagnosis of CNS lupus from radiographic findings alone, in the absence of clinical history. For example, it was often not possible to differentiate the focal white matter lesions seen in several of our patients from those found in patients with ischemic disease or multiple sclerosis. In a small number of patients, the MRI findings alone can suggest a diagnosis of vasculitis: for example, a young patient with multiple small gray and white matter lesions in multiple artery territories.

As noted in Patients and Methods, we used both $T_{1}$ - and $T_{2}$-weighted images for most patients. We believe this adequately covered the gamut of CNS pathologic findings. Other pulse sequences, such as inversion recovery, are unlikely to provide additional information. Scans in additional planes could have been performed (e.g., coronal or sagittal) for corroboration of equivocal findings, but this was generally not possible because of the time limitations.

Improved detection of the anatomic location of brain injury may assist in classifying NPSLE patients into appropriate subgroups for further study. MRI appears to provide a superior means of documenting, over time, the presence and course of cerebral injury in lupus. This technique may prove particularly valuable in assessing the outcome of therapeutic trials in neuropsychiatric lupus.

\section{REFERENCES}

1. Zvaifler N: Neurologic manifestations, The Clinical Management of Systemic Lupus Erythematosus. Edited by $P$ Schur. New York, Grune \& Stratton, 1983, pp 167-188

2. Small P, Mass MF, Kohler PF, Harbeck RJ: Central nervous system involvement in SLE: diagnostic profile and clinical features. Arthritis Rheum 20:869-878, 1977

3. Kassan SS, Lockshin MD: Central nervous system lupus erythematosus: the need for classification. Arthritis Rheum 22:1382-1385, 1979

4. Bresnihan B: CNS lupus. Clin Rheum Dis 8:183-195, 1982

5. Johnson RT, Richardson EP: The neurological manifestations of systemic lupus erythematosus: a clinicalpathological study of 24 cases and review of the literature. Medicine (Baltimore) 47:337-369, 1968

6. Ellis SF, Verity MA: Central nervous system involvement in systemic lupus erythematosus: a review of neuropsychiatric findings in 57 cases 1955-1977. Semin Arthritis Rheum 8:212-221, 1979

7. Gonzalez-Scarano F, Lisak RP, Bilaniuk LT, Zimmerman RA, Atkins PC, Zweiman B: Cranial computed 
tomography in the diagnosis of systemic lupus erythematosus. Ann Neurol 5:158-165, 1979

8. Bilaniuk LT, Patel S, Zimmerman RA: Computed tomography of systemic lupus erythematosus. Radiology 124: 119-121, 1977

9. Carette S, Urowitz M, Grossman H, St. Louis E: Cranial computerized tomography in systemic lupus erythematosus. J Rheumatol 9:855-859, 1982

10. Pinching AJ, Travers RL, Hughes GRV, Jones T, Moss S: Oxygen-15 brain scanning for detection of cerebral involvement in systemic lupus erythematosus. Lancet I:898-900, 1978

11. Grigor R, Edmonds J, Lewkonia R, Bresnihan B, Hughes GRV: Systemic lupus erythematosus: a prospective analysis. Ann Rheum Dis 37:121-128, 1979

12. Bennahum DA, Messner R, Shoop JD: Brain scan findings in central nervous system involvement by lupus erythematosus. Ann Intern Med 81:763-765, 1974

13. Gibson T, Myers AR: Nervous system involvement in systemic lupus erythematosus. Ann Rheum Dis 35:398406, 1976

14. Bradbury EM, Radda GK, Allen P: Nuclear magnetic resonance techniques in medicine. Ann Intern Med 98:514-529, 1983

15. Bradley WG, Waluch V, Yadley RA, Wycoff RR: Comparison of $C T$ and $M R$ in 400 patients with suspected disease of the brain and central spinal cord. Radiology 152:695-702, 1984

16. Bydder GM, Steiner RE, Young IR, Hall AS, Thomas DJ, Marshall J, Pallis CA, Legg NJ: Clinical NMR imaging of the brain: 140 cases. AJR 139:215-236, 1982

17. Johnson MA, Li DKB, Bryant DJ, Payne JA: Magnetic resonance imaging: serial observations in multiple sclerosis. AJNR 5:495-499, 1984

18. Aisen AA, Gabrielsen TO, McCune WJ: MR imaging of systemic lupus erythematosus involving the brain. AJNR 6:197-201, 1985

19. Vermess M, Bernstein RM, Bydder GM, Steiner RE, Young IR, Hughes GRV: Nuclear magnetic resonance (NMR) imaging of the brain in systemic lupus erythematosus. J Comput Assist Tomogr 7:461-467, 1983

20. Tan EM, Cohen AS, Fries JE, Masi AT, McShane DJ, Rothfield NF, Schaller JG, Talal N, Winchester RJ: The 1982 revised criteria for the classification of systemic lupus erythematosus. Arthritis Rheum 25:1271-1277, 1982

21. Kent JF, Fife EH: Precise standardization of reagents for complement fixation. Am J Trop Med Hyg 12:103116, 1963

22. Jado M, Patel T, Hughes CP, Danzinger W, Berg L: Brain atrophy in dementia judged by $\mathrm{CT}$ scan ranking. AJNR 4:499-500, 1983

23. Huckman MS, Fox IH, Topel J: The validity of criteria for the evaluation of cerebral atrophy by computed tomography. Radiology 116:85-92, 1975

24. Ostrov SG, Quencer RM, Goyks N, Autumn R: Cerebral atrophy in systemic lupus erythematosus: steroid or disease-induced phenomenon? AJNR 3:21-23, 1982

25. Bentson J, Reza M, Winter J, Wilson G: Steroids and apparent cerebral atrophy on computed tomography scans. J Comput Assist Tomogr 2:16-23, 1978 